\title{
Association of TERT polymorphisms with chronic hepatitis $B$ in a Chinese Han population
}

\author{
Guoxia Ren ${ }^{1,2, *}$, Xu Liu ${ }^{3, *}$, Zhendong Yư ${ }^{4}$, Jingjie $\mathbf{L i}^{5}$, Fanglin Niư ${ }^{5}$, Tianbo Jin ${ }^{5}$, Jikui \\ Liu $^{3}$ and Mingwei Chen ${ }^{1}$ \\ ${ }^{1}$ Department of Respiratory and Critical Care Medicine, The First Affiliated Hospital of School of Medicine of Xi'an Jiaotong \\ University, Xi'an 710061, China \\ ${ }^{2}$ Department of Intergrated Traditional Chinese and Western Medicine, Xi'an Chest Hospital, Xi'an 710100, China \\ ${ }^{3}$ Hepato-Pancreato-Biliary Surgery, Peking University Shenzhen Hospital, Guangdong, Shenzhen 518036, China \\ ${ }^{4}$ Central Laboratory, Peking University Shenzhen Hospital, Guangdong, Shenzhen 518036, China \\ ${ }^{5}$ Key Laboratory of Resource Biology and Biotechnology in Western China (Northwest University), Ministry of Education, \\ School of Life Sciences, Northwest University, Xi'an, Shaanxi 710069, China \\ *These authors contributed equally to this work and Joint first author \\ Correspondence to: Jikui Liu, email: liu8929@126.com \\ Mingwei Chen, email: chen_mingwei1964@163.com \\ Keywords: chronic hepatitis B; TERT; single nucleotide polymorphisms (SNPS); case-control; Chinese Han
}

Received: September 05, 2017 Accepted: December 26, $2017 \quad$ Published: January 03,2018

Copyright: Ren et al. This is an open-access article distributed under the terms of the Creative Commons Attribution License 3.0 (CC BY 3.0), which permits unrestricted use, distribution, and reproduction in any medium, provided the original author and source are credited.

\section{ABSTRACT}

In this study, we investigated the association between the polymorphisms of telomerase reverse transcriptase (TERT) gene and the risk of chronic hepatitis B (CHB) in a Chinese Han population. Four single nucleotide polymorphisms (SNPs) in TERT (rs10069690, rs2242652, rs2853677 and rs2853676) were genotyped from 224 CHB patients and $\mathbf{3 0 0}$ healthy controls using the Sequenom Mass-ARRAY platform. We used genetic model, haplotype analyses, chi-square test, logistic regression analysis to evaluate the association between SNPs and CHB risk. The relative risk was estimated by odd ratios (ORs) and $95 \%$ confidence intervals (CIs). We found that rs 10069690 was significantly associated with an increased CHB risk in the dominant model (adjusted OR $=1.70,95 \% \mathrm{CI}: 1.06-2.71, P=0.031$ ) and additive model (adjusted $O R=1.62$, 95\% CI: 1.09-2.41, $P=0.018$ ). The haplotype "TA" (rs10069690 and rs2242652) was found to be associated with an increased risk of CHB (adjusted OR $=1.58,95 \% \mathrm{CI}$ : 1.05-2.38, $P=0.027$ ). Our results suggested potential genetic contributes for TERT in CHB development in a Chinese Han population. Future functional and association studies with larger sample sizes are required to confirm these findings.

\section{INTRODUCTION}

Hepatitis B virus (HBV) infection is a serious public health problem worldwide, with approximately 2 billion people having a history of HBV infection and 350 million of them suffering from chronic HBV infection [1]. Based on a national epidemiological survey, it has been estimated that the weighted prevalence of hepatitis B surface antigen (HBsAg) was roughly $7.18 \%$ in Chinese population [2]. Although the host factors (such as infection age, gender and immune status), viral and environmental factors are thought to be involved in affecting the development of CHB. The mechanisms underlying the different clinical outcomes of HBV infection have not been fully understood. Studies indicated that host genetic factors play a critical role in the development of HBV infection, especially single nucleotide polymorphisms (SNPs), is regarded to be one of the determinants for this clinical heterogeneity $[3,4]$. Some polymorphisms have been reported to be involved in susceptibility to CHB in disease severity and progression, or disease prognosis $[4,5]$.

Telomeres, located at the ends of eukaryotic chromosomes, play a crucial role in maintaining the integrity of chromosome and stability of the genome $[6,7]$. Telomeres are approximately $10-15 \mathrm{~kb}$ in human somatic cells, and they shorten by 50-200 bp after each cycle of 
mitotic division [8]. In general, the incomplete replication of linear DNA molecules and gradual shortening of telomere length lead to regulated cell senescence and apoptosis or cell death. It has been reported that telomere length is associated with chronic liver diseases [9], CHB and hepatocellular carcinoma [10]. Interestingly, previous studies demonstrated that a number of polymorphisms in telomere biology gene (telomerase reverse transcriptase, TERT) are significantly associated with telomere length [11-13]. In addition, a recent report indicated the genotype TT of TERT polymorphism rs2736098 is associated with a decreased risk of CHB in Chinese males [14].

The TERT gene, located at human chromosome band 5 p15.33, consists of 16 exons and 15 introns spanning about $35 \mathrm{~kb}$. As the reverse transcriptase catalytic subunit of telomerase, the TERT is essential for the maintenance of telomere DNA length in chromosomes [15]. The activation of telomerase plays a key role in cellular immortalization and the malignant transformation of human cells. This activation requires the TERT catalyst [16]. A number of studies have also reported that variants of TERT gene are associated with a significantly higher susceptibility to several cancers [17-20]. However, to date, little study is reported on the association between genetic variations in the TERT gene and the risk of CHB. In this study, we conducted a case-control study consisting of $242 \mathrm{CHB}$ patients and 300 healthy controls to investigate the association between the four common genetic variants in TERT and the risk CHB in a Chinese Han population.

\section{RESULTS}

\section{Participants}

The basic characteristic of the participants are shown in Table 1, including gender, age, smoking and drinking. A total of $242 \mathrm{CHB}$ patients (188 males and 54 females) with a mean age of $50.04( \pm 12.048)$ years and 300 healthy controls (180 males and 120 females) with a mean age of $60.42( \pm 5.143)$ years were enrolled in the study. There were significant differences in gender, smoking, drinking and age distribution between the $\mathrm{CHB}$ cases and control groups $(P<0.05)$ (Table 1$)$. In order to eliminate those residual confounding effects, the variable of gender, smoking, drinking and age were adjusted in later multivariate unconditional logistic regression analysis.

\section{Allele distributions}

The detailed data of each SNP in TERT gene are shown in Table 2. Our data indicated that all four SNPs investigated were in Hardy-Weinberg equilibrium in the control subjects $(P>0.05)$. We compared the differences in frequency distributions of alleles between cases and controls by Chi-squared test. No associations were observed between the alleles of the four SNPs and CHB risk in the allele model. We also performed a Bonferroni correction and determined that none of the four SNPS showed statistical significant associations with $\mathrm{CHB}$ risk.

\section{Genetic models}

We further assessed the association between each SNP and CHB risk in an unconditional logistic regression analysis, which was performed using four models: genotype, dominant, recessive, and additive model (Table 3). The SNP rs 10069690 was found to be associated with an increased risk of $\mathrm{CHB}$ in the dominant model (OR $=$ 1.48, 95\% CI: $1.02-2.14, P=0.040$; adjusted $\mathrm{OR}=1.70$, 95\% CI: $1.06-2.71, P=0.031)$ both before and after the adjustment for age, gender, smoking and drinking; and in the additive model (adjusted OR $=1.62,95 \%$ CI: $1.09-2.41, P=0.018$ ) after the adjustment for age, gender, smoking and drinking. No significantly statistical associations were found under the other models.

\section{Haplotype}

The two SNPs rs10069690 and rs2242652 in the TERT gene showed significant linkage disequilibrium, as shown in Figure 1. In order to assess the associations between SNP haplotypes and CHB risk, a Wald test was performed using an unconditional multivariate regression analysis. The frequency of haplotype "TA" in the case group was significantly higher than that in the health control group. The haplotype "TA" was found to be associated with an increased risk of $\mathrm{CHB}$ (adjusted $\mathrm{OR}=$ $1.58,95 \% \mathrm{CI}: 1.05-2.38, P=0.027$ ) adjusted by gender, age, smoking and drinking, as shown in Table 4.

\section{DISCUSSION}

In this case-control study, we investigated the association between SNPs in the TERT gene and the risk of $\mathrm{CHB}$ in a Chinese Han population. The results showed that rs10069690 was significantly associated with an increased risk of CHB in the dominant model and additive model. In addition, we found that the haplotype "TA" (rs10069690 and rs2242652) was associated with an increased risk of $\mathrm{CHB}$.

TERT gene encodes the catalytic subunit of telomerase reverse transcriptase, which is really important component of telomerase. Several studies have reported that TERT regulates cell proliferation and metastasis, and that it also has a strong effect on alternative splicing and genetic control of telomere length $[21,22]$. The variants of the TERT has been found to be associated with many types of cancers and aging-related disorders, including glioma [17], lung cancer [19], breast cancer [12], gastric cancer [23], astrocytoma [18] and so on. It has been demonstrated that the genotype TT of TERT polymorphism rs 2736098 
Table 1: Basic characteristic of the participants

\begin{tabular}{ccccccc}
\hline \multirow{2}{*}{ Gender } & Characteristic & Case $(\boldsymbol{N}=\mathbf{2 4 2})$ & Frequency & Control $(\boldsymbol{N}=\mathbf{3 0 0})$ & Frequency & $\boldsymbol{P}$-value \\
\hline \multirow{2}{*}{ Smoking } & female & 54 & $22.3 \%$ & 120 & $40.0 \%$ & $<0.001$ \\
& male & 188 & $77.7 \%$ & 180 & $60.0 \%$ & \\
\multirow{2}{*}{ Drinking } & Yes & 126 & $52.1 \%$ & 89 & $29.7 \%$ & $<0.001$ \\
& No & 116 & $47.9 \%$ & 189 & $63.0 \%$ & \\
\multirow{2}{*}{ Age } & Yes & 90 & $37.2 \%$ & 79 & $26.3 \%$ & 0.033 \\
& No & 152 & $62.8 \%$ & 199 & $66.3 \%$ & \\
& years (mean $\pm \mathrm{SD})$ & $50.04 \pm 12.048$ & & $60.42 \pm 5.143$ & & $<0.001$ \\
\hline
\end{tabular}

SD: Standard deviation $P<0.05$ indicates statistical significant. The smoking and drinking information of the 22 cases in the control group were missing.

Table 2: Basic characteristic of the four SNPs in TERT

\begin{tabular}{|c|c|c|c|c|c|c|c|c|c|}
\hline \multirow{2}{*}{ SNP-ID } & \multirow{2}{*}{ Position } & \multirow{2}{*}{ Band } & \multirow{2}{*}{ Role } & \multirow{2}{*}{$\begin{array}{c}\text { Alleles } \\
\text { A/B }\end{array}$} & \multirow{2}{*}{ HWE } & \multicolumn{2}{|c|}{ MAF } & \multirow{2}{*}{ OR (95\% CI) } & \multirow{2}{*}{$P$} \\
\hline & & & & & & case & control & & \\
\hline rs10069690 & 1279790 & $5 \mathrm{p} 15.33$ & Intron & $\mathrm{T} / \mathrm{C}$ & 0.347 & 0.187 & 0.144 & $1.37(0.99-1.90)$ & 0.054 \\
\hline rs2242652 & 1280028 & $5 p 15.33$ & Intron & $\mathrm{A} / \mathrm{G}$ & 0.523 & 0.181 & 0.160 & $1.16(0.84-1.59)$ & 0.357 \\
\hline rs2853677 & 1287194 & $5 p 15.33$ & Intron & $\mathrm{G} / \mathrm{A}$ & 0.696 & 0.366 & 0.332 & $1.16(0.91-1.50)$ & 0.234 \\
\hline rs2853676 & 1288547 & $5 \mathrm{p} 15.33$ & Intron & $\mathrm{T} / \mathrm{C}$ & 0.817 & 0.158 & 0.147 & $1.09(0.78-1.52)$ & 0.611 \\
\hline
\end{tabular}

SNP: Single nucleotide polymorphism, A: Minor alleles, B: Major alleles, HWE: Hardy-Weinberg equilibrium, MAF: Minor allele frequency, OR: Odds ratio, 95\% CI: 95\% Confidence interval.

was associated with a decreased risk of CHB in Chinese males [14]. In this study, we found that TERT gene polymorphism rs10069690 and haplotype "TA" were associated with CHD risk in a Chinese Han population. We suggested that TERT gene may have association with cancers and disease by influencing the balancing the telomere length. In addition, Telomerase or TERT and telomeres have long been recognized to play pivotal parts in regulating immunological activity [24]. It is thus likely that TERT variants modify risk of CHB by influencing host immune function. However, the mechanistic details have not yet been elucidated.

Recently, it was reported that the $\mathrm{T}$ allele of rs10069690 was associated with an increased risk of breast cancer [12], lung cancer [19], esophageal cancer [23], gastric cancer [25], coronary heart disease [26]. However, rs 10069690 was found to be associated with significantly reduced risk of hepatocellular carcinoma, prostate cancer [27]. We didn't observe any association between the three SNPs (rs2242652, rs2853677and rs2853676) and CHD risk. Previous studies found that the three SNPs were also associated with several cancers risk [17, 23, 25, 28]. To date, there has been little report on the correlation between the polymorphisms of TERT and CHB risk. To our knowledge, this is the first report demonstrating an association between the variant rs 10069690 and CHB risk, which need to be confirmed in further studies.
It should be noted that this study has limitations. First, the sample size is not large enough, and the CHB patients and controls included only Chinese population lived in Shaanxi Province. Second, the functional assessment of TERT genetic variants was not performed. Finally, the potential influences of environmental factors on the results could not be completely eliminated. Therefore, future prospective studies are required to confirm these findings, and the interaction of genetic and environmental factors in the development of $\mathrm{CHB}$, and determine the functional role of these SNPs, with a larger sample.

In conclusion, our study identified that rs 10069690 and haplotype "TA" in TERT gene are associated with a significantly increased risk of $\mathrm{CHB}$, which may have the potentially to serve as prognostic biomarker for CHB in the Han Chinese population. Further validation of the functionality of the variants and its association with risk of $\mathrm{CHB}$ in other ethnic populations is warranted.

\section{MATERIALS AND METHODS}

\section{Participants}

A total of 542 unrelated Chinese Han individuals consisting of $242 \mathrm{CHB}$ patients and 300 healthy controls were recruited in the case-control study. All the patients were recruited from the Haikou people's Hospital, and 
Table 3: Genetic models analyses of the association between the SNPs and CHB risk

\begin{tabular}{|c|c|c|c|c|c|c|c|c|}
\hline SNP-ID & Model & Genotype & Case & Control & OR $(95 \%$ CI $)$ & $\boldsymbol{P}$ & OR $(95 \%$ CI $)$ & $P^{a}$ \\
\hline \multirow[t]{8}{*}{ rs 10069690} & Codominant & TT & 8 & 8 & $1.37(0.50-3.72)$ & 0.539 & $2.73(0.84-8.88)$ & 0.096 \\
\hline & & $\mathrm{TC}$ & 75 & 69 & $1.49(1.01-2.19)$ & 0.043 & $1.60(0.98-2.60)$ & 0.061 \\
\hline & & $\mathrm{CC}$ & 160 & 219 & 1.00 & - & 1.00 & - \\
\hline & Dominant & TT-TC & 83 & 77 & $1.48(1.02-2.14)$ & 0.040 & $1.70(1.06-2.71)$ & 0.027 \\
\hline & & $\mathrm{CC}$ & 160 & 219 & & & & \\
\hline & Recessive & TT & 8 & 8 & $1.23(0.45-3.32)$ & 0.689 & $2.39(0.74-7.72)$ & 0.144 \\
\hline & & TC-CC & 235 & 288 & & & & \\
\hline & Additive & --- & --- & --- & $1.37(0.99-1.89)$ & 0.057 & $1.62(1.09-2.41)$ & 0.018 \\
\hline \multirow[t]{8}{*}{ rs 2242652} & Codominant & AA & 8 & 9 & $1.16(0.44-3.08)$ & 0.763 & $2.05(0.64-6.55)$ & 0.225 \\
\hline & & AG & 72 & 78 & $1.21(0.83-1.76)$ & 0.333 & $1.22(0.76-1.98)$ & 0.415 \\
\hline & & GG & 163 & 213 & 1.00 & - & 1.00 & - \\
\hline & Dominant & AA-AG & 80 & 87 & $1.20(0.83-1.73)$ & 0.325 & $1.29(0.82-2.05)$ & 0.274 \\
\hline & & GG & 163 & 213 & & & & \\
\hline & Recessive & AA & 8 & 9 & $1.10(0.42-2.90)$ & 0.846 & $1.94(0.61-6.12)$ & 0.262 \\
\hline & & AG-GG & 235 & 291 & & & & \\
\hline & Additive & --- & --- & --- & $1.16(0.84-1.59)$ & 0.362 & $1.30(0.88-1.93)$ & 0.190 \\
\hline \multirow[t]{8}{*}{ rs2853677 } & Codominant & GG & 28 & 31 & $1.28(0.72-2.28)$ & 0.398 & $1.81(0.87-3.77)$ & 0.114 \\
\hline & & GA & 122 & 137 & $1.26(0.88-1.81)$ & 0.203 & $1.43(0.91-2.25)$ & 0.121 \\
\hline & & AA & 93 & 132 & 1.00 & - & 1.00 & - \\
\hline & Dominant & GG-GA & 150 & 168 & $1.27(0.90-1.79)$ & 0.178 & $1.49(0.97-2.31)$ & 0.070 \\
\hline & & AA & 93 & 132 & & & & \\
\hline & Recessive & GG & 28 & 31 & $1.13(0.66-1.94)$ & 0.658 & $1.49(0.75-2.98)$ & 0.256 \\
\hline & & GA-AA & 215 & 269 & & & & \\
\hline & Additive & --- & --- & --- & $1.18(0.91-1.52)$ & 0.222 & $1.37(0.99-1.91)$ & 0.058 \\
\hline \multirow[t]{8}{*}{ rs 2853676} & Codominant & $\mathrm{TT}$ & 3 & 7 & $0.55(0.14-2.17)$ & 0.395 & $1.42(0.26-7.73)$ & 0.683 \\
\hline & & $\mathrm{TC}$ & 71 & 74 & $1.24(0.84-1.81)$ & 0.277 & $1.23(0.76-1.98)$ & 0.398 \\
\hline & & $\mathrm{CC}$ & 170 & 219 & 1.00 & - & 1.00 & - \\
\hline & Dominant & TT-TC & 74 & 81 & $1.18(0.81-1.71)$ & 0.393 & $1.24(0.78-1.97)$ & 0.370 \\
\hline & & $\mathrm{CC}$ & 170 & 219 & & & & \\
\hline & Recessive & $\mathrm{TT}$ & 3 & 7 & $0.52(0.13-2.04)$ & 0.349 & $1.35(0.25-7.30)$ & 0.728 \\
\hline & & TC-CC & 241 & 293 & & & & \\
\hline & Additive & --- & --- & --- & $1.09(0.78-1.53)$ & 0.605 & $1.22(0.80-1.87)$ & 0.362 \\
\hline
\end{tabular}

SNP: Single nucleotide polymorphism, OR: Odds ratio, 95\% CI: 95\% Confidence interval aP were adjusted by age, gender, smoking and drinking. $P<0.05$ indicates statistical significant.

diagnosed as a clinical disease of $\mathrm{CHB}$ according to the diagnostic criteria of CHB based on history of hepatitis B virus (HBV) infection, $\mathrm{HBsAg}$ /anti-HBs, $\mathrm{HBeAg}$ /anti-HBe and anti-HBc serostatus, HBV DNA level, biochemical liver function, ultrasonography and/or computerized tomography (CT)/ magnetic resonance imaging (MRI) [29]. The patients with the family history of liver, renal, endocrine and cardiovascular disorders disease were excluded. The control subjects were randomly selected from physical examination center of the Haikou people's Hospital during the same period, with negativity for $\mathrm{HBsAg}, \mathrm{HBcAg}$, anti$\mathrm{HCV}$ and anti-HIV and with no abnormalities based on physical examination. All the subjects were Chinese Han individuals and their ancestors had lived in the region at least the three generations. A uniform questionnaire was used at enrollment including gender, age, smoking and drinking. The characteristics of the participants are shown in Table 1.

\section{Ethics statement}

The collected blood samples and the protocol in this study were strictly conformed to the Declaration of Helsinki and were approved by the institutional ethical committee of the Haikou people's Hospital. Written consents were obtained from all participants before participation in the study. 
Table 4: Haplotype frequencies and associated with CHB risk

\begin{tabular}{cccccc}
\hline SNPs & Haplotype & F_A & F_U & OR (95\% CI) & $\boldsymbol{P}$ \\
\hline rs10069690|rs2242652 & TA & 0.1777 & 0.1385 & $1.58(1.05-2.38)$ & 0.027 \\
& CG & 0.8161 & 0.8395 & $0.77(0.52-1.14)$ & 0.194 \\
\hline
\end{tabular}

F_A: Frequency in case, F_U: Frequency in control, OR: Odds ratio, 95\% CI: 95\% Confidence interval $P$-values were adjusted by gender, age, smoking and drinking. $P<0.05$ indicates statistical significance.

\section{Genotyping}

Five milliliters of peripheral venous blood was obtained from each subject and collected in an EDTA tube, and then the blood samples were stored in a refrigerator at $-20^{\circ} \mathrm{C}$ for DNA extraction. Genomic DNA was extracted from whole blood using the GoldMag-Mini Whole Blood Genomic DNA Purification Kit (GoldMag. Co. Ltd., Xi'an, China) based on the manufacturers' instructions. DNA concentration and purity were evaluated using a spectrophotometer (NanoDrop 2000; Thermo Fisher Scientific, Waltham, MA, USA) at wavelengths of A260 and A280nm.

We selected four SNPs (rs10069690, rs2242652, rs2853677 and rs2853676) from previously reported TERT gene polymorphisms [17, 19, 23, 25, 28], and matched SNPs with MAF $>5 \%$ in the HapMap of the Chinese Han Beijing population selected for association analysis. The sequences of amplification and extension primers corresponding to each SNP were designed by the Sequenom MassARRAY Assay Design 3.0 Software (Sequenom, San Diego, CA, USA), as showed in Table 5. Genotyping of the SNPs were performed using the Sequenom MassARRAY platform (Sequenom, San Diego, CA, USA) according to the standard protocol recommended by the manufacturer. We used the Sequenom Typer 4.0 software to perform data management and analyses.

\section{Statistical analysis}

All statistical analyses were performed using the SPSS 19.0 statistical software (SPSS, Chicago, IL) and Microsoft Excel. We used the Pearson's $\chi^{2}$ test and Welch's $t$ test to evaluate the differences in the demographic characteristics between the cases and controls. The Hardy-Weinberg equilibrium (HWE) of each SNP was assessed in order to compare the expected frequencies of the genotypes in the controls using $\chi^{2}$ test. The allele frequencies of the two groups were compared with chi-square test, and to evaluate associations between the SNPs and risk of CHB in the four models (genotype, dominant, recessive, and additive). Linkage disequilibrium analysis and SNP haplotypes were analyzed using the Haploview software package (version 4.2). The relative risk was estimated by odd ratios (ORs) and $95 \%$ confidence intervals (CIs) using unconditional logistic regression analysis. The $P$-value $<0.05$ was considered statistically significant and all statistical tests were two-sided.

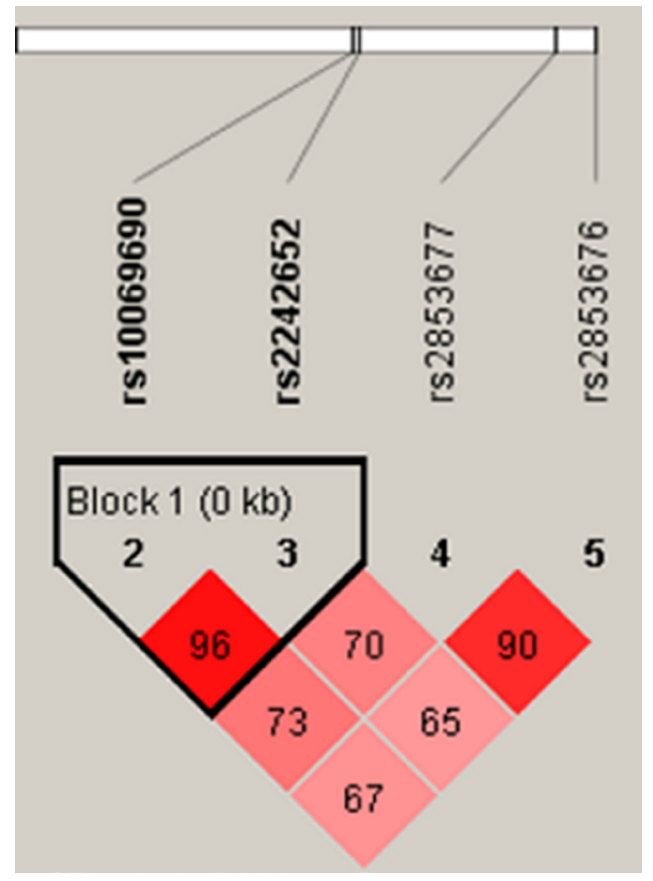

Figure 1: Haplotype block map for the four SNPs in TERT. The LD between each pair SNPs is standardized D', bright red corresponding to s very strong LD; white corresponding to no LD; pink corresponding to intermediate LD. 
Table 5: The sequences of primers of each SNP

\begin{tabular}{cccc}
\hline SNP-ID & 2nd-PCRP & 1st-PCRP & UEP \\
\hline rs10069690 & ACGTTGGATGATGTGTGTTGCACACGGGAT & ACGTTGGATGCCTGTGGCTGCGGTGGCTG & GGGATCCTCATGCCA \\
rs2242652 & ACGTTGGATGAGGCTCTGAGGACCACAAGA & ACGTTGGATGACAGCAGGACACGGATCCAG & gtcgGAGGACCACAAGAAGCAGC \\
rs2853677 & ACGTTGGATGGCAAGTGGAGAATCAGAGTG & ACGTTGGATGATCCAGTCTGACAGTCGTTG & gggtAATCAGAGTGCACCAG \\
rs2853676 & ACGTTGGATGCAAAACTAAGACCCAAGAGG & ACGTTGGATGTGTCTCCTGCTCTGAGACC & agatGGAAGTCTGACGAAGGC \\
\hline
\end{tabular}

SNP: Single nucleotide polymorphism; PCRP: Polymerase chain reaction primer; UEP: unique base extension primer Sequences are written in the $5^{\prime} \rightarrow 3^{\prime}$ (left to right) orientation.

\section{ACKNOWLEDGMENTS}

We are grateful to the all individuals for their participation in the study. We also thank the clinicians and hospital staff who contributed to sample and data collection for this study.

\section{CONFLICTS OF INTEREST}

The authors declare that there are no conflicts of interest.

\section{REFERENCES}

1. Trépo C, Chan HL, Lok A. Hepatitis B virus infection. Lancet. 2014; 384:2053-63.

2. Liang X, Bi S, Yang W, Wang L, Cui G, Cui F, Zhang Y, Liu J, Gong X, Chen Y, Wang F, Zheng H, Wang F, et al. Epidemiological serosurvey of hepatitis B in China-declining HBV prevalence due to hepatitis B vaccination. Vaccine. 2009; 27:6550-7. https://doi.org/10.1016/j. vaccine.2009.08.048.

3. He YL, Zhao YR, Zhang SL, Lin SM. Host susceptibility to persistent hepatitis B virus infection. World J Gastroenterol. 2006; 12:4788-93.

4. Liu L, Zhang J, Lu Y, Fang C, Li S, Lin J. Correlations between ASCC3 Gene Polymorphisms and Chronic Hepatitis B in a Chinese Han Population. Plos One. 2015; 10:e0141861.

5. Eskandari E, Metanat M, Pahlevani E, Nakhzari-Khodakheir T. Association between TGF $\beta 1$ polymorphisms and chronic hepatitis B infection in an Iranian population. 2017; 50.

6. Blackburn EH. Telomeres and telomerase: the means to the end (Nobel lecture). Angew Chem Int Ed Engl. 2010; 49:7405-21. https://doi.org/10.1002/anie.201002387.

7. Pfeiffer V, Lingner J. Replication of telomeres and the regulation of telomerase. Cold Spring Harb Perspect Biol. 2013; 5:a010405. https://doi.org/10.1101/cshperspect. a010405.

8. Kipling D. Telomeres, replicative senescence and human ageing. Maturitas. 2001; 38:25-37; discussion -8.

9. Donati B, Valenti L. Telomeres, NAFLD, Chronic Liver Disease. Int J Mol Sci. 2016; 17:383. https://doi. org/10.3390/ijms17030383.

10. Ma Q, Cai J, Cai Y, Xu Y, Chang F, Xu L, Zhang G, Guo X. Association of telomere length in peripheral leukocytes with chronic hepatitis B and hepatocellular carcinoma. Medicine (Baltimore). 2016; 95:e4970. https://doi.org/10.1097/ MD.0000000000004970.

11. Codd V, Nelson CP, Albrecht E, Mangino M, Deelen J, Buxton JL, Hottenga JJ, Fischer K, Esko T, Surakka I. Identification of seven loci affecting mean telomere length and their association with disease. Nature Genetics. 2013; 45:1-2.

12. Bojesen SE, Pooley KA, Johnatty SE, Beesley J, Michailidou K, Tyrer JP, Edwards SL, Pickett HA, Shen HC, Smart CE. Multiple independent variants at the TERT locus are associated with telomere length and risks of breast and ovarian cancer. Nature Genetics. 2013; 45:371-84.

13. Do SK, Yoo SS, Choi YY, Choi JE, Jeon HS, Lee WK, Lee SY, Lee J, Cha SI, Kim CH. Replication of the results of genome-wide and candidate gene association studies on telomere length in a Korean population. Korean J Intern Med. 2015; 30:719-26.

14. Cheng G, Yuan X, Wang F, Sun Q, Xin Q, Li K, Sun C, Lin Z, Luan Y, Xu Y. Association Between the Telomerase rs2736098 TT Genotype and a Lower Risk of Chronic Hepatitis B, Cirrhosis in Chinese Males. Clinical \& Translational Gastroenterology. 2017; 8:e79.

15. Young NS. Telomere biology and telomere diseases: implications for practice and research. Hematology. 2010; 2010:30.

16. Zhang A, Zheng C, Lindvall C, Hou M, Ekedahl J, Lewensohn R, Yan Z, Yang X, Henriksson M, Blennow E. Frequent amplification of the telomerase reverse transcriptase gene in human tumors. Cancer Research. 2000; 60:6230-5.

17. Li G, Jin TB, Wei XB, He SM, Liang HJ, Yang HX, Cui Y, Chen C, Cai LB, Gao GD. Selected polymorphisms of GSTP1 and TERT were associated with glioma risk in Han Chinese. Cancer Epidemiology. 2012; 36:525.

18. Jin TB, Zhang JY, Li G, Du SL, Geng TT, Gao J, Liu QP, Gao GD, Kang LL, Chen C. RTEL1 and TERT polymorphisms are associated with astrocytoma risk in the Chinese Han population. Tumor Biology. 2013; 34:3659-66.

19. Gao L, Thakur A, Liang Y, Zhang S, Wang T, Chen T, Meng J, Wang L, Wu F, Jin T. Polymorphisms in the TERT gene are associated with lung cancer risk in the Chinese Han population. European Journal of Cancer Prevention the Official Journal of the European Cancer Prevention Organisation. 2014; 23:497-501. 
20. Ding $Y$, Du S, Sun P, Niu H, Duan M, Jin T, Yao H. Association between the TERT rs2736100 polymorphism and lung cancer risk: evidence from a case-control study and a meta-analysis.

21. Melin BS, Nordfjäll K, Andersson U, Roos G. hTERT cancer risk genotypes are associated with telomere length. Genetic Epidemiology. 2012; 36:368.

22. Hou L, Zhang X, Gawron AJ, Liu J. Surrogate tissue telomere length and cancer risk: shorter or longer? Cancer Letters. 2012; 319:130-5.

23. Wu Y, Yan M, Jing L, Li J, Chen Z, Peng C, Li B, Chen F, Jin T, Chao C. Genetic polymorphisms in TERT are associated with increased risk of esophageal cancer. Oncotarget. 2017; 8:10523-10530. https://doi.org/10.18632/oncotarget.14451.

24. Weng N. Telomeres and immune competency. Current Opinion in Immunology. 2012; 24:470-5.

25. Duan X, Cao W, Wang L, Liu S, Liu Z, Zhang B, Yang $\mathrm{H}$, Feng T, Zhang J, Zhang X. Genetic variants in TERT are associated with risk of gastric cancer in a Chinese Han population. Oncotarget. 2016; 7:82727-82732. https://doi. org/10.18632/oncotarget.13102.

26. Han H, Zhang J, Hou J, Wang H, Zheng J, Wang H, Zhong Z, Wang Y, Wang X, Yang B, Wang L, Quan D, Li J. Association of TERT polymorphisms and risk of coronary heart disease in a Chinese Han population. Oncotarget. 2017; 8:67519-67525. https://doi.org/10.18632/oncotarget.18727.

27. Wu D, Yu H, Sun J, Qi J, Liu Q, Li R, Zheng SL, Xu J, Kang J. Association of genetic polymorphisms in the telomerase reverse transcriptase gene with prostate cancer aggressiveness. Molecular Medicine Reports. 2015; 12:489.

28. Sheng X, Tong N, Tao G, Luo D, Wang M, Fang Y, Li J, Xu M, Zhang Z, Wu D. TERT polymorphisms modify the risk of acute lymphoblastic leukemia in Chinese children. Carcinogenesis. 2013; 34:228-35.

29. Lok ASF, Mcmahon BJ. Chronic hepatitis B. Hepatology. 2007; 45:507-39. 